\title{
An Open Cultural Digital Content Infrastructure
}

Ioanna-Ourania Stathopoulou, Haris Georgiadis, Vangelis Banos, Panagiotis Stathopoulos, Nikos Houssos, Evi Sachini

National Documentation Centre / National Hellenic Research Foundation Athens, Greece

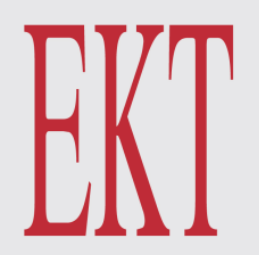

EONIKO KENTPO

TEKMHPI $\Omega \Sigma H \Sigma$

N A T I O N A L

DOCUMENTATION

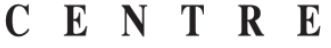




\section{Outline}

- Objective and scope

- Approach and design choices

- Architecture and implementation

- Related work

- Status and future work 


\section{Context and objectives of the activity}

- The funding environment

- More than 70 digital cultural heritage projects / about 60 million Euros

- Co-funding by Greece and EU structural funds

- Assist the funder to ensure the availability and quality of project output

- Availability of the produced material (metadata and digital files) at a central, secure, enterprise-grade infrastructure

- Infrastructure and mechanisms to check the quality of metadata and digital content generated through the projects 


\section{A suite of services for repositories}

- Harvesting and aggregation of content

- Single point of access to content / unified search and browse

- Validation (metadata + digital files)

- Largely automated checking of compliance with specifications

- Safe-keeping of digital files in the highest available quality 


\section{Why validation?}

- Problems identified in project output of past funding programmes (indicative list):

- Inadequate quality of metadata records - use of custom data models and formats instead of standard schemata

- Poor digitization quality, lack of basic features like OCR for text material

- Lack of standard programmatic interfaces for continuous access to the material

- Inadequate infrastructure to ensure availability and safekeeping

- Result: Low reuse and return on investment 


\section{Overview of the validation approach}

- Publish interoperability specifications in advance

- Associate successful validation with funding cash flows

- Validate multiple times throughout project - first during initial stages to provide early feedback

- Validation of both metadata and digital files

- Validation is largely automated - essential for scalability, feasibility and sustainability

- Validation of live systems

- The material is validated by directly retrieving it through programmatic interfaces (e.g. OAI-PMH)

- Modular and extensible validation infrastructure gradual support of multiple schemata, formats 


\section{Interoperability requirements}

- Specifications have been published in advance, before the beginning of the projects

- Available at http://hdl.handle.net/10442/8887

- Cover interoperability at the system, syntactic/structure, semantic levels

- Availability of the metadata as linked data is mandatory

- The funded institution is required to provide also information such as the detailed specifications of the primary cataloguing schema used, mappings to standard formats, the controlled vocabularies / thesauri utilised 


\section{System architecture}

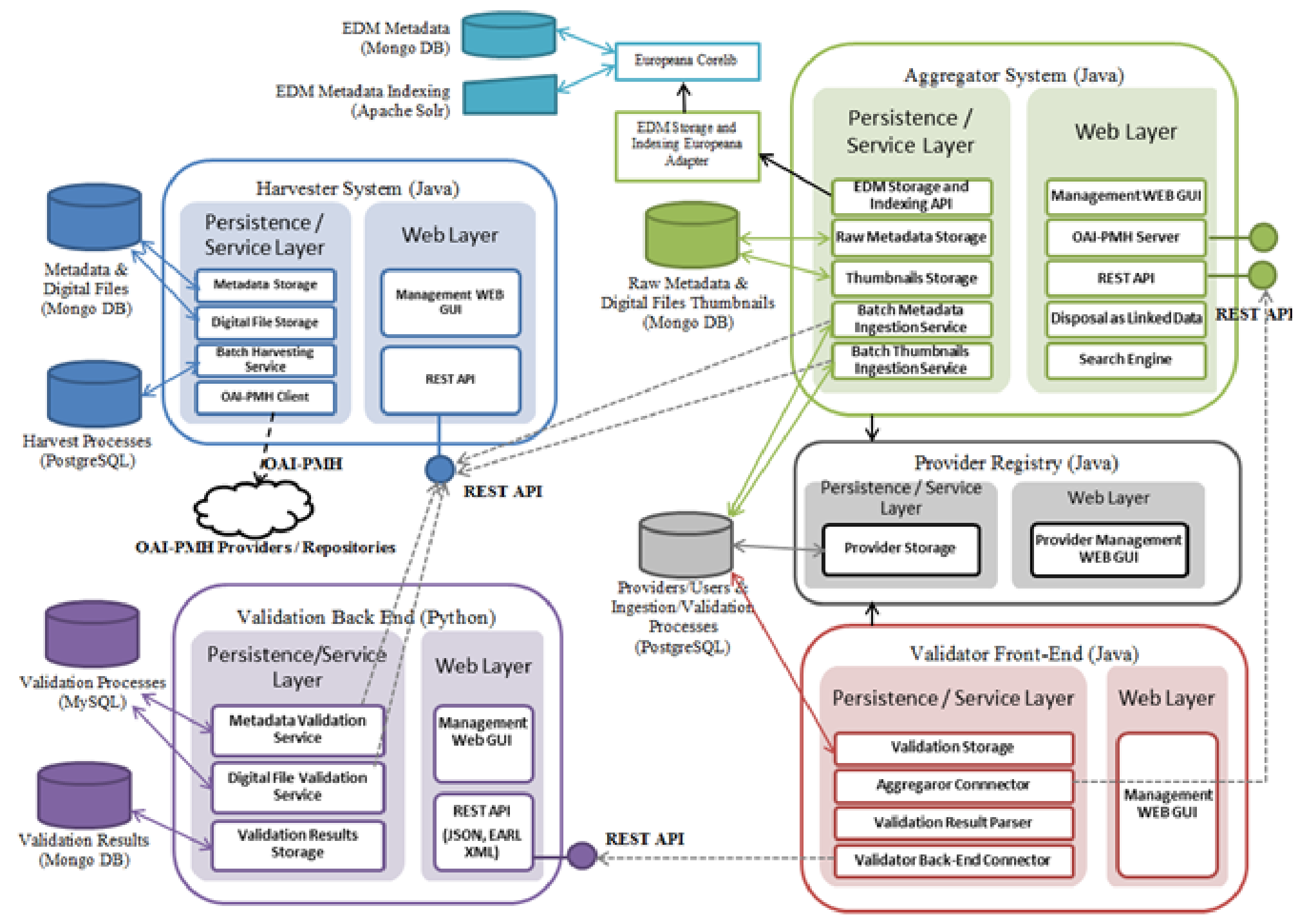

Digital Libraries Conference, 09-12 September 2014, London, UK 


\section{Data model and implementation}

\section{aspects}

- The aggregator uses the Europeana Data Model (EDM) as the main data model and metadata schema

- Infrastructure implemented using certain components of the Europeana open source software infrastructure

- A range of utilised technologies: Java, Python, REST APIs for interactions among components, Solr, MongoDB, PostgreSQL 


\section{Validation workflow}

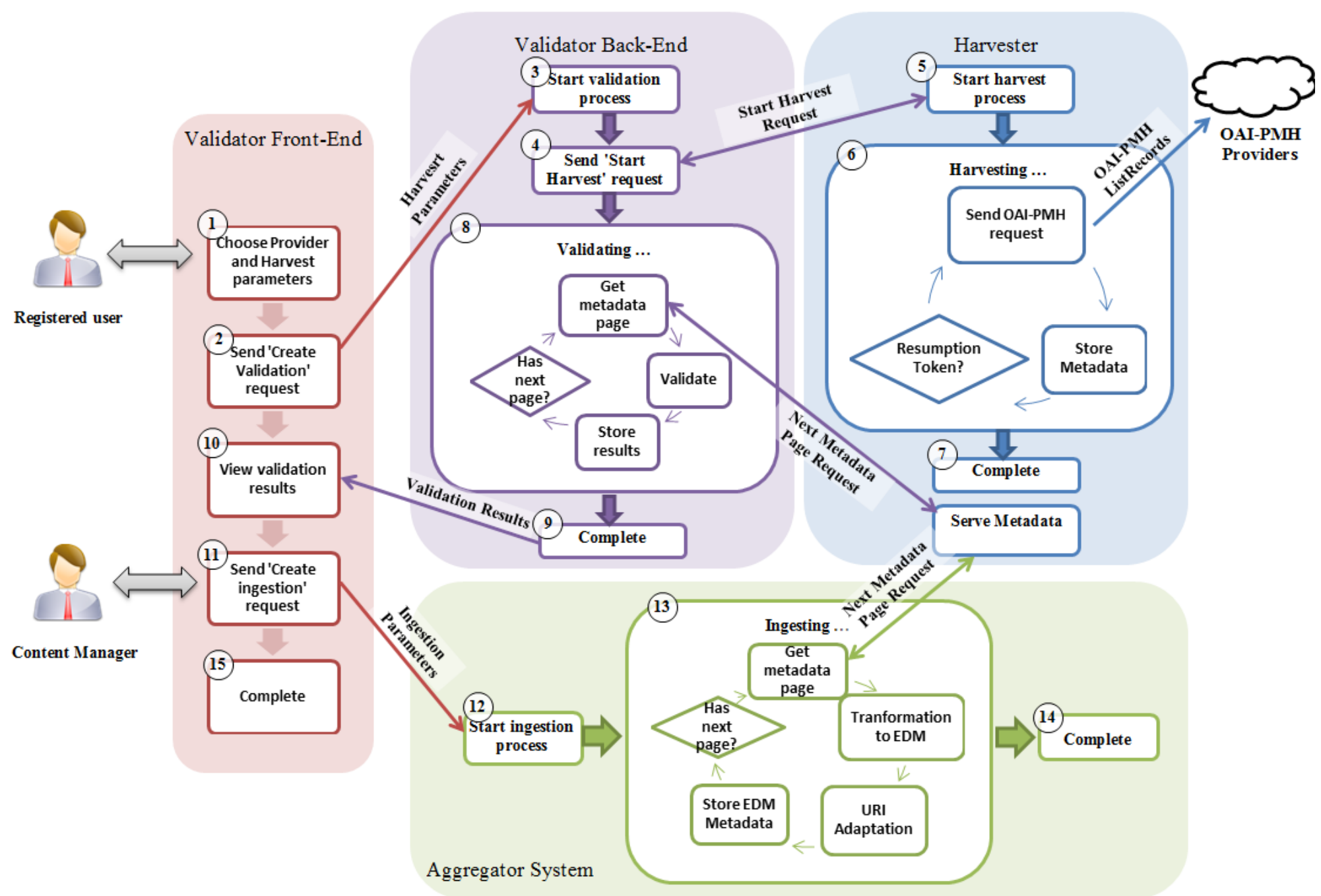




\section{Validation logic architecture and implementation}

- Two interacting components

- Front-end and back-end

- Front-end provides UI for authorized users to carry out validations / get results / produce reports, back-end provides the core validation logic

- Modular and extensible scheme for the definition and insertion of rules in the system

- Validation Domain-Specific Language (VDSL)

- Rules and rule-sets, boolean operators, control flow

- Simple JSON format

- Gradual support for checking compliance with various schemata and formats 


\section{Comparison with related work}

- Validators exists in various international systems (e.g. OpenAIRE, ARIADNE)

- Distinct features of our approach:

- Validation of both metadata (records, controlled vocabularies) and digital files

- Extensive validation at the semantic level

- Modularity and extensibility allows combined validation along multiple dimensions (e.g. check availability of metadata in multiple formats)

- Validation Domain Specific Language

- Support for connecting validation with administrative procedures in a decoupled fashion 


\section{Status of implementation and operation - further work}

- The infrastructure is in production since Spring 2014

- Several validations have been already completed with the infrastructure

- Next steps:

- Provide a user interface for repository managers to perform test validations before actually submitting their content

- Refine validation rules and aspects of their implementation

- Public operation of the aggregator portal 


\section{Acknowledgements}

- The work presented in this article has been partly supported by the project

- "Platform for provision of services for deposit, management and dissemination of Open Public Data and Digital Content" (Ref No 327378)

- Co-funded by Greece and the European UnionEuropean Regional Development Fund through the Operational Programme "Digital

Convergence" (NSFR) 


\title{
Thank you for your attention!
}

- More info:

\author{
http://www.epset.gr/en/SaaS Services
}

http://www.epset.gr/en/SaaS Services/Interoperability -Testing

iostath, hgeorgiadis, vbanos, pstath, nhoussos, esachin AT ekt.gr 